\title{
On derived equivalences for selfinjective algebras
}

\author{
Hiroki Abe and Mitsuo Hoshino
}

\begin{abstract}
We show that if $A$ is a representation-finite selfinjective artin algebra then every $P^{\bullet} \in \mathrm{K}^{\mathrm{b}}\left(\mathcal{P}_{A}\right)$ with $\operatorname{Hom}_{\mathrm{K}(\operatorname{Mod}-A)}\left(P^{\bullet}, P^{\bullet}[i]\right)=0$ for $i \neq 0$ and $\operatorname{add}\left(P^{\bullet}\right)=\operatorname{add}\left(\nu P^{\bullet}\right)$ is a direct summand of a tilting complex, and that if $A, B$ are derived equivalent representation-finite selfinjective artin algebras then there exists a sequence of selfinjective artin algebras $A=B_{0}, B_{1}, \cdots, B_{m}=B$ such that, for any $0 \leq i<m, B_{i+1}$ is the endomorphism algebra of a tilting complex for $B_{i}$ of length $\leq 1$.
\end{abstract}

Let $A$ be an artin algebra. Rickard [16, Proposition 9.3] showed that for any tilting complex $P^{\bullet} \in \mathrm{K}^{\mathrm{b}}\left(\mathcal{P}_{A}\right)$ the number of nonisomorphic indecomposabele direct summands of $P^{\bullet}$ coincides with the rank of $K_{0}(A)$, the Grothendieck group of $A$, which generalizes earlier results [6, Proposition 3.2] and [15, Theorem 1.19]. He raised a question whether a complex $P^{\bullet} \in \mathrm{K}^{\mathrm{b}}\left(\mathcal{P}_{A}\right)$ with $\operatorname{Hom}_{\mathrm{K}(\operatorname{Mod}-A)}\left(P^{\bullet}, P^{\bullet}[i]\right)$ $=0$ for $i \neq 0$ is a tilting complex or not if the number of nonisomorphic indecomposable direct summands of $P^{\bullet}$ coincides with the rank of $K_{0}(A)$ (see also [15]). In case $P^{\bullet}$ is a projective resolution of a module $T \in \bmod -A$ with proj $\operatorname{dim} T_{A} \leq 1$, Bongartz [4, Lemma of 2.1] has settled the question affirmatively. More precisely, he showed that every $T \in \bmod -A$ with proj $\operatorname{dim} T_{A} \leq 1$ and $\operatorname{Ext}_{A}^{1}(T, T)=0$ is a direct summand of a classical tilting module, i.e., a tilting module of projective dimension $\leq 1$. Unfortunetely, this is not true in general (see [16, Section 8]). Our first aim of this note is to show that if $A$ is a representation-finite selfinjective artin algebra then every $P^{\bullet} \in \mathrm{K}^{\mathrm{b}}\left(\mathcal{P}_{A}\right)$ with $\operatorname{Hom}_{\mathrm{K}(\operatorname{Mod}-A)}\left(P^{\bullet}, P^{\bullet}[i]\right)=0$ for $i \neq 0$ and $\operatorname{add}\left(P^{\bullet}\right)=\operatorname{add}\left(\nu P^{\bullet}\right)$, where $\nu$ is the Nakayama functor, is a direct summand of a tilting complex (Theorem 3.6).

Rickard [17, Theorem 4.2] showed that the Brauer tree algebras over a field with the same numerical invariants are derived equivalent to each other. Subsequently, Okuyama pointed out that for any Brauer tree algebras $A, B$ with the same numerical invariants there exists a sequence of Brauer tree algebras

2000 Mathematics Subject Classification: Primary: 18E30, 18G35; Secondary: 16G30, $18 \mathrm{E} 40$.

Key words: derived equivalence, tilting complex, torsion theory, selfinjective algebra. 
$A=B_{0}, B_{1}, \cdots, B_{m}=B$ such that, for any $0 \leq i<m, B_{i+1}$ is the endomorphism algebra of a tilting complex for $B_{i}$ of length $\leq 1$. These facts can be formulated as follows. For any tilting complex $P^{\bullet} \in \mathrm{K}^{\mathrm{b}}\left(\mathcal{P}_{A}\right)$ associated with a certain sequence of idempotents in a ring $A$, there exists a sequence of rings $A=B_{0}, B_{1}, \cdots, B_{m}=\operatorname{End}_{\mathrm{K}(\operatorname{Mod}-A)}\left(P^{\bullet}\right)$ such that, for any $0 \leq i<m, B_{i+1}$ is the endomorphism ring of a tilting complex for $B_{i}$ of length $\leq 1$ determined by an idempotent (see [11, Proposition 3.2]). We refer to [7], [14] for other examples of derived equivalences which are iterations of derived equivalences induced by tilting complexes of length $\leq 1$. Our second aim of this note is to show that for any derived equivalent representation-finite selfinjective artin algebras $A, B$ there exists a sequence of selfinjective artin algebras $A=B_{0}, B_{1}, \cdots, B_{m}=B$ such that, for any $0 \leq i<m, B_{i+1}$ is the endomorphism algebra of a tilting complex for $B_{i}$ of length $\leq 1$ (Theorem 3.7).

For a ring $A$, we denote by $\operatorname{Mod}-A$ the category of right $A$-modules. We denote by $A^{\mathrm{op}}$ the opposite ring of $A$ and consider left $A$-modules as right $A^{\mathrm{op}}$ modules. Sometimes, we use the notation $X_{A}$ (resp., ${ }_{A} X$ ) to stress that the module $X$ considered is a right (resp., left) $A$-module. For an object $X$ in an additive category $\mathcal{B}$, we denote by $\operatorname{add}(X)$ the full subcategory of $\mathcal{B}$ whose objects are direct summands of finite direct sums of copies of $X$ and by $X^{(n)}$ the direct sum of $n$ copies of $X$. For a cochain complex $X^{\bullet}$ over an abelian category $\mathcal{A}$, we denote by $\mathrm{Z}^{n}\left(X^{\bullet}\right), \mathrm{Z}^{\prime n}\left(X^{\bullet}\right)$ and $\mathrm{H}^{n}\left(X^{\bullet}\right)$ the $n$-th cycle, the $n$-th cocycle and the $n$-th cohomology of $X \bullet$, respectively. For an additive category $\mathcal{B}$, we denote by $\mathrm{K}(\mathcal{B})$ (resp., $\mathrm{K}^{+}(\mathcal{B}), \mathrm{K}^{-}(\mathcal{B}), \mathrm{K}^{\mathrm{b}}(\mathcal{B})$ ) the homotopy category of complexes (resp., bounded below complexes, bounded above complexes, bounded complexes) over $\mathcal{B}$. As usual, we consider objects of $\mathcal{B}$ as complexes over $\mathcal{B}$ concentrated in degree zero. For an abelian category $\mathcal{A}$, we denote by $\mathrm{D}(\mathcal{A})$ (resp., $\left.\mathrm{D}^{+}(\mathcal{A}), \mathrm{D}^{-}(\mathcal{A}), \mathrm{D}^{\mathrm{b}}(\mathcal{A})\right)$ the derived category of complexes (resp., bounded below complexes, bounded above complexes, bounded complexes) over $\mathcal{A}$. We always consider $\mathrm{K}^{*}(\mathcal{B})$ (resp., $\mathrm{D}^{*}(\mathcal{A})$ ) as a full triangulated subcategory of $\mathrm{K}(\mathcal{B})$ (resp., $\mathrm{D}(\mathcal{A})$ ), where $*=+,-$ or $\mathrm{b}$. We denote by $\operatorname{Hom}^{\bullet}(-,-)$ the associated single complex of the double hom complex.

We refer to [3], [8], [19] for basic results in the theory of derived categories and to [16], [18] for definitions and basic properties of derived equivalences and tilting complexes.

\section{Preliminaries}

Throughout this note, $R$ is a commutative artinian ring with the Jacobson radical $\mathfrak{m}$ and $A$ is an $\operatorname{artin} R$-algebra, i.e., $A$ is a ring endowed with a ring homomorphism $R \rightarrow A$ whose image is contained in the center of $A$ and is finitely generated as an $R$-module.

For any artin $R$-algebra $A$, we denote by mod- $A$ the full subcategory of Mod- $A$ consisting of finitely generated modules and by $\mathcal{P}_{A}$ (resp., $\mathcal{I}_{A}$ ) the full subcategory of mod- $A$ consisting of projective (resp., injective) modules. Also, we set $D=\operatorname{Hom}_{R}(-, E(R / \mathfrak{m}))$, where $E(R / \mathfrak{m})$ is an injective envelope of $R / \mathfrak{m}$ 
in Mod- $R$, and $\nu=D \circ \operatorname{Hom}_{A}(-, A)$, which is called the Nakayama functor.

Remark 1.1. The Krull-Schmidt theorem holds in mod- $A$, i.e., for any nonzero module $X \in \bmod -A$ the following hold.

(1) $X$ decomposes into a direct sum of indecomposable submodules.

(2) $X$ is indecomposable if and only if $\operatorname{End}_{A}(X)$ is local.

Remark 1.2. The following hold.

(1) $X \stackrel{\sim}{\rightarrow} D^{2} X, x \mapsto(h \mapsto h(x))$, for all $X \in \bmod -R$.

(2) $D: \bmod -A \rightarrow \bmod -A^{\text {op }}$ is an anti-equivalence and induces anti-equivalences $\mathcal{P}_{A} \stackrel{\sim}{\rightarrow} \mathcal{I}_{A^{\text {op }}}$ and $\mathcal{I}_{A} \stackrel{\sim}{\rightarrow} \mathcal{P}_{A^{\text {op }}}$.

(3) $\nu: \bmod -A \rightarrow \bmod -A$ induces an equivalence $\mathcal{P}_{A} \stackrel{\sim}{\rightarrow} \mathcal{I}_{A}$.

Lemma 1.3. For any $P^{\bullet} \in \mathrm{K}^{\mathrm{b}}\left(\mathcal{P}_{A}\right)$ the following are equivalent.

(1) $P^{\bullet} \in \operatorname{add}\left(\nu P^{\bullet}\right)$.

(2) $\nu P^{\bullet} \in \operatorname{add}\left(P^{\bullet}\right)$.

(3) $\operatorname{add}\left(P^{\bullet}\right)=\operatorname{add}\left(\nu P^{\bullet}\right)$.

Proof. Note that every idempotent splits in K(Mod- $A$ ) (see [3, Proposition 3.2]). Thus, since we have an isomorphism of artin $R$-algebras

$$
\operatorname{End}_{\mathrm{K}(\operatorname{Mod}-A)}\left(P^{\bullet}\right) \simeq \operatorname{End}_{\mathrm{K}(\operatorname{Mod}-A)}\left(\nu P^{\bullet}\right),
$$

it follows that $P^{\bullet}$ and $\nu P^{\bullet}$ have the same number of nonisomorphic indecomposable direct summands.

Recall that $A$ is said to be selfinjective if the equivalent conditions of Lemma 1.3 are satisfied for $P^{\bullet}=A$.

Remark 1.4. If $A$ is selfinjective, then $\nu: \bmod -A \rightarrow \bmod -A$ is an equivalence and induces an equivalence $\mathcal{P}_{A} \stackrel{\sim}{\rightarrow} \mathcal{P}_{A}$.

Lemma 1.5 ([10, Lemma 3.1]). For any $P^{\bullet} \in \mathrm{K}^{\mathrm{b}}\left(\mathcal{P}_{A}\right)$ and $X^{\bullet} \in \mathrm{K}(\operatorname{Mod}-A)$ we have a bifunctorial isomorphism

$$
\operatorname{Hom}_{\mathcal{K}(\operatorname{Mod}-A)}\left(X^{\bullet}, \nu P^{\bullet}\right) \simeq D \operatorname{Hom}_{\mathrm{K}(\operatorname{Mod}-A)}\left(P^{\bullet}, X^{\bullet}\right) .
$$

Definition 1.6. For any $P^{\bullet} \in \mathrm{K}^{\mathrm{b}}\left(\mathcal{P}_{A}\right)$ we denote by $\mathcal{C}\left(P^{\bullet}\right)$ the full subcategory of $\mathrm{D}^{-}($Mod- $A)$ consisting of complexes $X^{\bullet}$ with $\operatorname{Hom}_{\mathrm{D}(\operatorname{Mod}-A)}\left(P^{\bullet}, X^{\bullet}[i]\right)=0$ for $i \neq 0$.

Lemma 1.7. Assume $A$ is selfinjective. Then for any tilting complex $P^{\bullet} \in$ $\mathrm{K}^{\mathrm{b}}\left(\mathcal{P}_{A}\right)$ the following are equivalent.

(1) $\operatorname{End}_{\mathrm{K}(\operatorname{Mod}-A)}\left(P^{\bullet}\right)$ is selfinjective. 
(2) $P^{\bullet} \in \mathcal{C}\left(\nu P^{\bullet}\right)$.

(3) $\operatorname{add}\left(P^{\bullet}\right)=\operatorname{add}\left(\nu P^{\bullet}\right)$.

Proof. Set $B=\operatorname{End}_{\mathrm{K}(\operatorname{Mod}-A)}\left(P^{\bullet}\right)$. Note that by Lemma $1.5 \nu P^{\bullet} \in \mathcal{C}\left(P^{\bullet}\right)$ and $\operatorname{Hom}_{\mathrm{K}(\operatorname{Mod}-A)}\left(P^{\bullet}, \nu P^{\bullet}\right) \simeq D\left({ }_{B} B\right)$.

$(1) \Leftrightarrow(3)$. Note first that we have an equivalence (see $[16$, Section 4$]$ )

$$
\operatorname{Hom}_{\mathrm{D}(\operatorname{Mod}-A)}\left(P^{\bullet},-\right): \mathcal{C}\left(P^{\bullet}\right) \stackrel{\sim}{\rightarrow} \operatorname{Mod}-B .
$$

We may consider $\operatorname{add}\left(P^{\bullet}\right)$ and $\operatorname{add}\left(\nu P^{\bullet}\right)$ as full subcategories of $\mathcal{C}\left(P^{\bullet}\right)$ via the canonical functor $\mathrm{K}^{\mathrm{b}}\left(\mathcal{P}_{A}\right) \rightarrow \mathrm{D}^{-}(\operatorname{Mod}-A)$. Then $\operatorname{add}\left(P^{\bullet}\right)$ and $\operatorname{add}\left(\nu P^{\bullet}\right)$ are closed under direct summands because every idempotent splits in $\mathrm{K}^{\mathrm{b}}\left(\mathcal{P}_{A}\right)$ (see [3, Proposition 3.4]). Thus the equivalence above induces equivalences $\operatorname{add}\left(P^{\bullet}\right) \stackrel{\sim}{\rightarrow} \mathcal{P}_{B}$ and $\operatorname{add}\left(\nu P^{\bullet}\right) \stackrel{\sim}{\rightarrow} \mathcal{I}_{B}$.

$(2) \Rightarrow(3)$. We have $\operatorname{Hom}_{\mathrm{K}(\operatorname{Mod}-A)}\left(P^{\bullet} \oplus \nu P^{\bullet},\left(P^{\bullet} \oplus \nu P^{\bullet}\right)[i]\right)=0$ for $i \neq 0$ and hence by $\left[11\right.$, Lemma 1.8] $\operatorname{add}\left(P^{\bullet}\right)=\operatorname{add}\left(\nu P^{\bullet}\right)$.

$(3) \Rightarrow(2)$. Obvious.

In case $A, B$ are finite dimentional selfinjective algebras over a field and $F: \mathrm{K}^{\mathrm{b}}\left(\mathcal{P}_{A}\right) \stackrel{\sim}{\rightarrow} \mathrm{K}^{\mathrm{b}}\left(\mathcal{P}_{B}\right)$ is an equivalence of triangulated categories, it was pointed out in [1, Section 2] that for any $P^{\bullet} \in \mathrm{K}^{\mathrm{b}}\left(\mathcal{P}_{A}\right)$ there exists an objectwise isomorphism $F\left(\nu P^{\bullet}\right) \simeq \nu F\left(P^{\bullet}\right)$. We need to extend this fact to the case of artin algebras.

Lemma 1.8. Let $A, B$ be derived equivalent selfinjective artin $R$-algebras and $F: \mathrm{K}^{\mathrm{b}}\left(\mathcal{P}_{A}\right) \stackrel{\sim}{\rightarrow} \mathrm{K}^{\mathrm{b}}\left(\mathcal{P}_{B}\right)$ an equivalence of triangulated categories. Then for any $P^{\bullet} \in \mathrm{K}^{\mathrm{b}}\left(\mathcal{P}_{A}\right)$ we have a functorial isomorphism $\nu F\left(P^{\bullet}\right) \simeq F\left(\nu P^{\bullet}\right)$.

Proof. Let $G: \mathrm{K}^{\mathrm{b}}\left(\mathcal{P}_{B}\right) \stackrel{\sim}{\rightarrow} \mathrm{K}^{\mathrm{b}}\left(\mathcal{P}_{A}\right)$ be a quasi-inverse of $F$. Then for any $P^{\bullet} \in$ $\mathrm{K}^{\mathrm{b}}\left(\mathcal{P}_{A}\right)$ and $Q^{\bullet} \in \mathrm{K}^{\mathrm{b}}\left(\mathcal{P}_{B}\right)$, by Lemma 1.5 we have bifunctorial isomorphisms

$$
\begin{aligned}
\operatorname{Hom}_{\mathrm{K}(\operatorname{Mod}-B)}\left(Q^{\bullet}, \nu F\left(P^{\bullet}\right)\right) & \simeq D \operatorname{Hom}_{\mathrm{K}(\operatorname{Mod}-B)}\left(F\left(P^{\bullet}\right), Q^{\bullet}\right) \\
& \simeq D \operatorname{Hom}_{\mathrm{K}(\operatorname{Mod}-A)}\left(P^{\bullet}, G\left(Q^{\bullet}\right)\right) \\
& \simeq \operatorname{Hom}_{\mathrm{K}(\operatorname{Mod}-A)}\left(G\left(Q^{\bullet}\right), \nu P^{\bullet}\right) \\
& \simeq \operatorname{Hom}_{\mathrm{K}(\operatorname{Mod}-B)}\left(Q^{\bullet}, F\left(\nu P^{\bullet}\right)\right) .
\end{aligned}
$$

The assertion follows by Yoneda lemma.

Definition 1.9. For any nonzero $P^{\bullet} \in \mathrm{K}^{-}\left(\mathcal{P}_{A}\right)$ we set

$$
a\left(P^{\bullet}\right)=\max \left\{i \in \mathbb{Z} \mid \mathrm{H}^{i}\left(P^{\bullet}\right) \neq 0\right\},
$$

and for any nonzero $P^{\bullet} \in \mathrm{K}^{+}\left(\mathcal{P}_{A}\right)$ we set

$$
b\left(P^{\bullet}\right)=\min \left\{i \in \mathbb{Z} \mid \operatorname{Hom}_{\mathrm{K}(\operatorname{Mod}-A)}\left(P^{\bullet}[i], A\right) \neq 0\right\} .
$$

Then for any nonzero $P^{\bullet} \in \mathrm{K}^{\mathrm{b}}\left(\mathcal{P}_{A}\right)$ we set $l\left(P^{\bullet}\right)=a\left(P^{\bullet}\right)-b\left(P^{\bullet}\right)$ and call it the length of $P^{\bullet}$. For the sake of convenience, we set $l\left(P^{\bullet}\right)=0$ for $P^{\bullet} \in \mathrm{K}^{\mathrm{b}}\left(\mathcal{P}_{A}\right)$ with $P^{\bullet} \simeq 0$. 
Remark 1.10 ([6]). For any complex $X^{\bullet}$ and $n \in \mathbb{Z}$ we define truncations

$$
\begin{aligned}
& \sigma_{\leq n}\left(X^{\bullet}\right): \cdots \rightarrow X^{n-2} \rightarrow X^{n-1} \rightarrow \mathrm{Z}^{n}\left(X^{\bullet}\right) \rightarrow 0 \rightarrow \cdots, \\
& \sigma_{\geq n}^{\prime}\left(X^{\bullet}\right): \cdots \rightarrow 0 \rightarrow \mathrm{Z}^{\prime n}\left(X^{\bullet}\right) \rightarrow X^{n+1} \rightarrow X^{n+2} \rightarrow \cdots .
\end{aligned}
$$

Then $P^{\bullet} \simeq \sigma_{\leq a}\left(P^{\bullet}\right)$ for any nonzero $P^{\bullet} \in \mathrm{K}^{-}\left(\mathcal{P}_{A}\right)$, where $a=a\left(P^{\bullet}\right)$, and $P^{\bullet} \simeq \sigma_{\geq b}^{\prime}\left(P^{\bullet}\right)$ for any nonzero $P^{\bullet} \in \mathrm{K}^{+}\left(\mathcal{P}_{A}\right)$, where $b=b\left(P^{\bullet}\right)$.

\section{Torsion theories}

We need to recall several definitions and basic results on torsion theories.

Definition $2.1([3])$. A pair $(\mathcal{T}, \mathcal{F})$ of full subcategories $\mathcal{T}, \mathcal{F}$ in an abelian category $\mathcal{A}$ is said to be a torsion theory for $\mathcal{A}$ if the following conditions are satisfied:

(1) $\mathcal{T} \cap \mathcal{F}=\{0\}$;

(2) $\mathcal{T}$ is closed under factor objects;

(3) $\mathcal{F}$ is closed under subobjects; and

(4) for any $X \in \mathcal{A}$ there exists an exact sequence $0 \rightarrow X^{\prime} \rightarrow X \rightarrow X^{\prime \prime} \rightarrow 0$ with $X^{\prime} \in \mathcal{T}$ and $X^{\prime \prime} \in \mathcal{F}$.

Definition 2.2. Let $\mathcal{A}$ be an abelian category and $\mathcal{C}$ a full subcategory of $\mathcal{A}$. Then we denote by ${ }^{\perp} \mathcal{C}$ (resp., $\mathcal{C}^{\perp}$ ) the full subcategory of $\mathcal{A}$ consisting of objects $X$ with $\operatorname{Hom}_{\mathcal{A}}(X, \mathcal{C})=0$ (resp., $\operatorname{Hom}_{\mathcal{A}}(\mathcal{C}, X)=0$ ). For an object $Y \in \mathcal{A}$, we use the notation ${ }^{\perp} Y$ (resp., $\left.Y^{\perp}\right)$ instead of ${ }^{\perp} \operatorname{add}(Y)\left(\right.$ resp., $\left.\operatorname{add}(Y)^{\perp}\right)$.

Remark 2.3. Let $(\mathcal{T}, \mathcal{F})$ be a torsion theory for an abelian category $\mathcal{A}$. Then the following hold.

(1) $\mathcal{F}=\mathcal{T}^{\perp}$ and $\mathcal{T}={ }^{\perp} \mathcal{F}$.

(2) $\mathcal{T}$ and $\mathcal{F}$ are closed under extensions.

(3) There exists a subfunctor $t$ of the identity functor $\mathbf{1}_{\mathcal{A}}: \mathcal{A} \rightarrow \mathcal{A}$, called the associated torsion radical, such that $t(X) \in \mathcal{T}$ and $X / t(X) \in \mathcal{F}$ for all $X \in \mathcal{A}$.

Proof. (1) By the conditions (1)-(3), $\mathcal{F} \subset \mathcal{T}^{\perp}$ and $\mathcal{T} \subset{ }^{\perp} \mathcal{F}$. On the other hand, by the condition (4), $\mathcal{T}^{\perp} \subset \mathcal{F}$ and ${ }^{\perp} \mathcal{F} \subset \mathcal{T}$.

(2) Immediate by (1).

(3) For each $X \in \mathcal{A}$, take an exact sequence

$$
0 \rightarrow X^{\prime} \stackrel{\iota x}{\longrightarrow} X \stackrel{\pi_{X}}{\longrightarrow} X^{\prime \prime} \rightarrow 0
$$

with $X^{\prime} \in \mathcal{T}$ and $X^{\prime \prime} \in \mathcal{F}$. For any $Z \in \mathcal{T}$, since $\operatorname{Hom}_{\mathcal{A}}\left(Z, X^{\prime \prime}\right)=0$, $\operatorname{Hom}_{\mathcal{A}}\left(Z, \iota_{X}\right)$ is an isomorphism. It follows that $X^{\prime}$ is maximum in the collection of subobjects of $X$ belonging to $\mathcal{T}$. We set $t(X)=X^{\prime}$. Next, let 
$f: X \rightarrow Y$ be a morphism. Since $\operatorname{Hom}_{\mathcal{A}}\left(X^{\prime}, Y^{\prime \prime}\right)=0, \pi_{Y} \circ f \circ \iota_{X}=0$ and there exists a unique morphism $f^{\prime}: X^{\prime} \rightarrow Y^{\prime}$ such that $f \circ \iota_{X}=\iota_{Y} \circ f^{\prime}$. We set $t(f)=f^{\prime}$. Then for any $X \in \mathcal{A}$ we have $\operatorname{id}_{X} \circ \iota_{X}=\iota_{X} \circ \operatorname{id}_{t(X)}$ and hence $t\left(\operatorname{id}_{X}\right)=\operatorname{id}_{t(X)}$. Also, for any consecutive morphisms $f: X \rightarrow Y$ and $g: Y \rightarrow Z$, since $f \circ \iota_{X}=\iota_{Y} \circ t(f)$ and $g \circ \iota_{Y}=\iota_{Z} \circ t(g)$, we have $g \circ f \circ \iota_{X}=\iota_{Z} \circ t(g) \circ t(f)$ and hence $t(g \circ f)=t(g) \circ t(f)$.

Although the next lemma is well-known, we include a proof because it will play an indispensable role in the next section.

Lemma 2.4. For any $Y \in \bmod -A$, by setting $\mathcal{T}={ }^{\perp} Y$ and $\mathcal{F}=\mathcal{T}^{\perp}$, we have a torsion theory $(\mathcal{T}, \mathcal{F})$ for $\bmod -A$.

Proof. It is obvious that the conditions (1)-(3) of Definition 2.1 are satisfied. Let $X \in \bmod -A$. Let $\left\{X_{\lambda}\right\}_{\lambda \in \Lambda}$ be the set of submodules of $X$ belonging to $\mathcal{T}$ and set $X^{\prime}=\bigcup_{\lambda \in \Lambda} X_{\lambda}$. Note that $\mathcal{T}$ is closed under extensions and finite direct sums. In particular, $\Lambda$ is directed, where $\lambda \leq \mu$ if and only if $X_{\lambda} \subset X_{\mu}$, and $X^{\prime}$ is a submodule of $X$. Thus we have an epimorphism $\bigoplus_{\lambda \in \Lambda} X_{\lambda} \rightarrow X^{\prime}$ in Mod- $A$ and, since $\operatorname{Hom}_{A}\left(\bigoplus_{\lambda \in \Lambda} X_{\lambda}, Y\right) \simeq \prod_{\lambda \in \Lambda} \operatorname{Hom}_{A}\left(X_{\lambda}, Y\right)=0$, it follows that $X^{\prime} \in \mathcal{T}$. Next, we claim that $X / X^{\prime} \in \mathcal{F}$. Let $Z \in \mathcal{T}$ and $f \in \operatorname{Hom}_{A}\left(Z, X / X^{\prime}\right)$. Take a pull-back of $f$ along with the canonical epimorphism $X \rightarrow X / X^{\prime}$ :

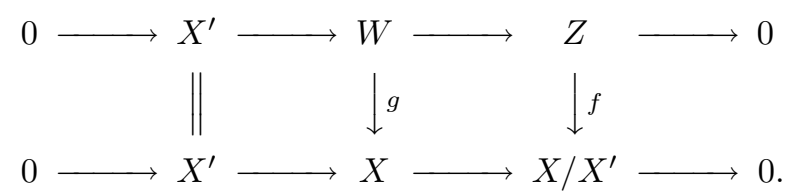

Then, since $W \in \mathcal{T}, \operatorname{Im} g \subset X^{\prime}$ and $f=0$.

Definition 2.5. Let $\mathcal{A}$ be an abelian category and $\mathcal{C}$ a full subcategory of $\mathcal{A}$ closed under extensions. Then an object $X \in \mathcal{C}$ is said to be Ext-projective (resp., Ext-injective) if $\operatorname{Ext}_{\mathcal{A}}^{1}(X, \mathcal{C})=0$ (resp., $\left.\operatorname{Ext}_{\mathcal{A}}^{1}(\mathcal{C}, X)=0\right)$.

Lemma 2.6. Let $(\mathcal{T}, \mathcal{F})$ be a torsion theory for mod- $A$. Then a module $X \in \mathcal{T}$ is Ext-injective if and only if $X=t(E)$ with $E$ an injective envelope of $X$.

Proof. "If" part. Let $E \in \bmod -A$ be an injective module and take an exact sequence

$$
0 \rightarrow t(E) \stackrel{\mu}{\rightarrow} Y \stackrel{\varepsilon}{\rightarrow} Z \rightarrow 0
$$

with $Z \in \mathcal{T}$. We claim that $\mu$ is a split monomorphism. Denote by $\iota: t(E) \rightarrow E$ the inclusion. By the injectivity of $E, \iota=\phi \circ \mu$ for some $\phi: Y \rightarrow E$. Note that by Remark 2.3(2) $Y \in \mathcal{T}$. Thus $\phi(Y) \subset t(E)$ and $\phi=\iota \circ \phi^{\prime}$ for some $\phi^{\prime}: Y \rightarrow t(E)$. Then $\iota=\iota \circ \phi^{\prime} \circ \mu$ and $\operatorname{id}_{t(E)}=\phi^{\prime} \circ \mu$. It follows that $t(E)$ is Ext-injective.

"Only if" part. Let $X \in \mathcal{T}$ and $E$ an injective envelope of $X$. We consider $X$ as a submodule of $E$. Then $X \subset t(E)$ and we have an exact sequence

$$
0 \rightarrow X \stackrel{\iota}{\rightarrow} t(E) \rightarrow t(E) / X \rightarrow 0
$$


Since $t(E) / X \in \mathcal{T}$, and since $X$ is Ext-injective, the inclusion $\iota: X \rightarrow t(E)$ has to be a split monomorphism. On the other hand, $E$ and hence $t(E)$ are essential extensions of $X$. It follows that $X=t(E)$.

We refer to [2, Chapter V, Sections 1 and 2] for the following Definitions 2.7, 2.8 and Lemmas 2.9, 2.11.

Definition 2.7. Let $\mathcal{A}$ be an abelian category and $\mathcal{C}$ a full subcategory of $\mathcal{A}$. Let $f: X \rightarrow Y$ be a morphism with $X, Y \in \mathcal{C}$. Then $f$ is said to be right (resp., left) almost split in $\mathcal{C}$ if $f$ is not a split epimorphism (resp., monomorphism) and if every morphism $h: Z \rightarrow Y$ (resp., $h: X \rightarrow Z$ ) with $Z \in \mathcal{C}$ factors through $f$ unless $h$ is a split epimorphism (resp., monomorphism).

Definition 2.8. Let $\mathcal{A}$ be an abelian category and $\mathcal{C}$ a full subcategory of $\mathcal{A}$ closed under extensions. Then a nonsplit exact sequence

$$
0 \rightarrow Z \stackrel{g}{\rightarrow} Y \stackrel{f}{\rightarrow} X \rightarrow 0
$$

with $X, Z \in \mathcal{C}$ is said to be an almost split sequence in $\mathcal{C}$ if the following conditions are satisfied:

(1) $\operatorname{End}_{\mathcal{A}}(X)$ and $\operatorname{End}_{\mathcal{A}}(Z)$ are local; and

(2) $f$ (resp., $g$ ) is right (resp., left) almost split in $\mathcal{C}$.

Lemma 2.9. Let $\mathcal{A}$ be an abelian category and $\mathcal{C}$ a full subcategory of $\mathcal{A}$ closed under extensions. Let

$$
0 \rightarrow Z_{1} \rightarrow Y_{1} \rightarrow X_{1} \rightarrow 0, \quad 0 \rightarrow Z_{2} \rightarrow Y_{2} \rightarrow X_{2} \rightarrow 0
$$

be almost split sequences in $\mathcal{C}$. Then $X_{1} \simeq X_{2}$ if and only if $Z_{1} \simeq Z_{2}$.

Definition 2.10. For each indecomposable module $X \in \bmod -A$, we take a minimal projective resolution $P_{X}^{\bullet} \rightarrow X$ and set $\tau X=\mathrm{Z}^{-1}\left(\nu P_{X}^{\bullet}\right)$.

Lemma 2.11. Let $X \in \bmod -A$ be an indecomposable nonprojective module. Then $\operatorname{Ext}_{A}^{1}(X, \tau X) \neq 0$ and the following hold.

(1) As a right module over $\operatorname{End}_{A}(X), \operatorname{Ext}_{A}^{1}(X, \tau X)$ is embedded in $D \operatorname{End}_{A}(X)$ and hence has a simple socle.

(2) A nonsplit exact sequence

$$
0 \rightarrow \tau X \rightarrow Y \rightarrow X \rightarrow 0
$$

representing a nonzero element of the socle of $\operatorname{Ext}_{A}^{1}(X, \tau X)$ is an almost split sequence in $\bmod -A$.

Lemma 2.12 ([9, Lemma 2]). Let $(\mathcal{T}, \mathcal{F})$ be a torsion theory for mod- $A$ and $X \in \mathcal{T}$ an indecomposable module. Then the following hold. 
(1) $X$ is Ext-projective if and only if $\tau X \in \mathcal{F}$.

(2) Assume $X$ is not Ext-projective and let $0 \rightarrow \tau X \rightarrow Y \rightarrow X \rightarrow 0$ be an almost split sequence in $\bmod -A$. Then the induced sequence

$$
0 \rightarrow t(\tau X) \rightarrow t(Y) \rightarrow X \rightarrow 0
$$

is an almost split sequence in $\mathcal{T}$.

Definition 2.13. Assume $A$ is selfinjective and let $\left\{e_{1}, \cdots, e_{n}\right\}$ be a basic set of orthogonal local idempotents in $A$. Then there exists a permutation $\rho$ of the set $I=\{1, \cdots, n\}$, called the Nakayama permutation, such that $\nu\left(e_{i} A\right) \simeq e_{\rho(i)} A$ for all $i \in I$.

Proposition 2.14. Assume $A$ is selfinjective and has a cyclic Nakayama permutation. Then for any tilting complex $P^{\bullet} \in \mathrm{K}^{\mathrm{b}}\left(\mathcal{P}_{A}\right)$ with $\operatorname{End}_{\mathrm{K}(\operatorname{Mod}-A)}\left(P^{\bullet}\right)$ selfinjective we have $l\left(P^{\bullet}\right)=0$.

Proof. Set $l=l\left(P^{\bullet}\right)$. We may assume $P^{i}=0$ unless $0 \leq i \leq l$. Suppose to the contrary that $l \geq 1$. Set $X=\mathrm{H}^{l}\left(P^{\bullet}\right)$ and $Y=\mathrm{H}^{0}\left(P^{\bullet}\right)$. Since by Lemma 1.7 $\operatorname{add}\left(P^{\bullet}\right)=\operatorname{add}\left(\nu P^{\bullet}\right)$, we have $\operatorname{add}\left(P^{\bullet}\right)=\operatorname{add}\left(\nu^{k} P^{\bullet}\right)$ for all $k \geq 0$. Thus for any $k \geq 0$, since $\operatorname{Hom}_{\mathrm{K}(\operatorname{Mod}-A)}\left(P^{\bullet}, P^{\bullet}[-l]\right)=0$, and since $\nu^{k} P^{\bullet} \in \operatorname{add}\left(P^{\bullet}\right)$, we have

$$
\begin{aligned}
\operatorname{Hom}_{A}\left(\nu^{k} X, Y\right) & \simeq \operatorname{Hom}_{A}\left(\mathrm{H}^{l}\left(\nu^{k} P^{\bullet}\right), \mathrm{H}^{0}\left(P^{\bullet}\right)\right) \\
& \simeq \operatorname{Hom}_{\mathrm{K}(\text { Mod- } A)}\left(\nu^{k} P^{\bullet}, P^{\bullet}[-l]\right) \\
& =0 .
\end{aligned}
$$

By Lemma 2.4 there exists a torsion theory $(\mathcal{T}, \mathcal{F})$ for mod- $A$ such that $\mathcal{T}={ }^{\perp} Y$ and $\mathcal{F}=\mathcal{T}^{\perp}$. Let $\left\{e_{1}, \cdots, e_{n}\right\}$ be a basic set of orthogonal local idempotents in $A$ and set $S_{i}=e_{i} A / e_{i} J$ for $1 \leq i \leq n$, where $J$ is the Jacobson radical of $A$. Note that $\nu S_{i} \simeq S_{\rho(i)}$ for all $1 \leq i \leq n$. Let $S \in \bmod -A$ be a simple module which is a factor module of $X$. For any $k \geq 0$, since $\nu^{k} X \in \mathcal{T}$, and since $\nu^{k} S$ is a facotr module of $\nu^{k} X$, we have $\nu^{k} S \in \mathcal{T}$. Note that $S \simeq S_{i}$ for some $1 \leq i \leq n$. Then $\nu^{k} S \simeq S_{\rho^{k}(i)}$ for all $k \geq 0$. Since $\rho$ is cyclic, it follows that $S_{i} \in \mathcal{T}$ for all $1 \leq i \leq n$. Thus $\mathcal{F}$ does not contain any simple module and $\mathcal{F}=\{0\}$. On the other hand, by the construction we have $0 \neq Y \in \mathcal{F}$, a contradiction.

\section{Main results}

To begin with, we modify [4, Lemma of 2.1] as follows.

Lemma 3.1. Let $P^{\bullet} \in \mathrm{K}^{\mathrm{b}}\left(\mathcal{P}_{A}\right)$ be a complex with $\operatorname{Hom}_{\mathrm{K}(\text { Mod-A) }}\left(P^{\bullet}, P^{\bullet}[i]\right)=0$ for $i \neq 0$ and $\operatorname{add}\left(P^{\bullet}\right)=\operatorname{add}\left(\nu P^{\bullet}\right)$. Assume there exists a tilting complex $T^{\bullet} \in \mathrm{K}^{\mathrm{b}}\left(\mathcal{P}_{A}\right)$ such that $\operatorname{Hom}_{\mathrm{K}(\operatorname{Mod}-A)}\left(P^{\bullet}, T^{\bullet}[i]\right)=0$ unless $-1 \leq i \leq 0$. Form a distinguished triangle in $\mathrm{K}^{\mathrm{b}}\left(\mathcal{P}_{A}\right)$

$$
Q^{\bullet} \rightarrow P^{\bullet(n)} \stackrel{f}{\rightarrow} T^{\bullet} \rightarrow
$$

such that $\operatorname{Hom}_{\mathrm{K}(\operatorname{Mod}-A)}\left(P^{\bullet}, f\right)$ is epic. Then $Q^{\bullet} \oplus P^{\bullet}$ is a tilting complex. 
Proof. Note first that such a homomorphism $f$ exists. To see this, set $X^{\bullet}=$ $\operatorname{Hom}_{A}^{\bullet}\left(P^{\bullet}, T^{\bullet}\right) \in \mathrm{K}^{\mathrm{b}}(\bmod -R)$. Then $\operatorname{Hom}_{\mathrm{K}(\operatorname{Mod}-A)}\left(P^{\bullet}, T^{\bullet}\right) \simeq \mathrm{H}^{0}\left(X^{\bullet}\right) \in \bmod -R$, i.e., $\operatorname{Hom}_{\mathrm{K}(\operatorname{Mod}-A)}\left(P^{\bullet}, T^{\bullet}\right)$ is finitely generated over $R$. It then follows that $\operatorname{Hom}_{\mathrm{K}(\operatorname{Mod}-A)}\left(P^{\bullet}, T^{\bullet}\right)$ is finitely generated over $\operatorname{End}_{\mathrm{K}(\operatorname{Mod}-A)}\left(P^{\bullet}\right)$. Take a set of generators $f_{1}, \cdots, f_{n} \in \operatorname{Hom}_{\mathrm{K}(\operatorname{Mod}-A)}\left(P^{\bullet}, T^{\bullet}\right)$ over $\operatorname{End}_{\mathrm{K}(\operatorname{Mod}-A)}\left(P^{\bullet}\right)$ and set

$$
f=\left(f_{1}, \cdots, f_{n}\right): P^{\bullet(n)} \rightarrow T^{\bullet} .
$$

It then follows by the construction that $\operatorname{Hom}_{\mathrm{K}(\operatorname{Mod}-A)}\left(P^{\bullet}, f\right)$ is epic.

Obviously, $\operatorname{add}\left(Q^{\bullet} \oplus P^{\bullet}\right)$ generates $\mathrm{K}^{\mathrm{b}}\left(\mathcal{P}_{A}\right)$ as a triangulated category. Note also that by Lemma $1.5 \operatorname{Hom}_{\mathrm{K}(\operatorname{Mod}-A)}\left(T^{\bullet}, P^{\bullet}[i]\right)=0$ unless $0 \leq i \leq 1$.

Claim: The following hold.

(1) $\operatorname{Hom}_{\mathrm{K}(\operatorname{Mod}-A)}\left(P^{\bullet}, Q^{\bullet}[i]\right)=0$ for $i \neq 0$.

(2) $\operatorname{Hom}_{\mathrm{K}(\operatorname{Mod}-A)}\left(Q^{\bullet}, P^{\bullet}[i]\right)=0$ for $i \neq 0$.

(3) $\operatorname{Hom}_{\mathrm{K}(\operatorname{Mod}-A)}\left(T^{\bullet}, Q^{\bullet}[i]\right)=0$ for $i>1$.

(4) $\operatorname{Hom}_{\mathrm{K}(\operatorname{Mod}-A)}\left(Q^{\bullet}, T^{\bullet}[i]\right)=0$ for $i<-1$.

Proof. (1), (3) and (4) follow by the construction and (2) follows by (1) and Lemma 1.5.

Now, by (1), (3) of Claim $\operatorname{Hom}_{\mathrm{K}(\operatorname{Mod}-A)}\left(Q^{\bullet}, Q^{\bullet}[i]\right)=0$ for $i>0$ and by (2), (4) of Claim $\operatorname{Hom}_{\mathrm{K}(\operatorname{Mod}-A)}\left(Q^{\bullet}, Q^{\bullet}[i]\right)=0$ for $i<0$. This finishes the proof of Lemma 3.1

Corollary 3.2. Assume $A$ is selfinjective. Let $P^{\bullet} \in \mathrm{K}^{\mathrm{b}}\left(\mathcal{P}_{A}\right)$ be a complex with $P^{i}=0$ unless $0 \leq i \leq 1$. Assume $\operatorname{Hom}_{\mathrm{K}(\operatorname{Mod}-A)}\left(P^{\bullet}, P^{\bullet}[i]\right)=0$ for $i \neq 0$ and $\operatorname{add}\left(P^{\bullet}\right)=\operatorname{add}\left(\nu P^{\bullet}\right)$. Then there exists some $Q^{\bullet} \in \mathrm{K}^{\mathrm{b}}\left(\mathcal{P}_{A}\right)$ such that $Q^{\bullet} \oplus P^{\bullet}$ is a tilting complex. In particular, if the number of nonisomorphic indecomposable direct summands of $P^{\bullet}$ coincides with the rank of the Grothendieck group $K_{0}(A)$, then $P^{\bullet}$ is a tilting complex.

Proof. Applying Lemma 3.1 to $T^{\bullet}=A$, the first assertion follows. The last assertion follows by [16, Proposition 9.3].

Recall that $A$ is said to be representation-finite if there exist only a finite number of nonisomorphic indecomposable modules in mod- $A$.

Remark 3.3 ([13] and [17]). Let $A, B$ be derived equivalent selfinjective artin $R$-algebras. Then $A$ is representation-finite if and only if so is $B$.

Proof. This follows by the fact that $A, B$ are stably equivalent (see $[13$, Theorem $3.8]$ and [17, Corollary 2.2]). 
Lemma 3.4. Assume $A$ is selfinjective and representation-finite. Let $P^{\bullet} \in$ $\mathrm{K}^{\mathrm{b}}\left(\mathcal{P}_{A}\right)$ be a complex of length $\geq 1$ with $\operatorname{Hom}_{\mathrm{K}(\operatorname{Mod}-A)}\left(P^{\bullet}, P^{\bullet}[i]\right)=0$ for $i \neq 0$ and $\operatorname{add}\left(P^{\bullet}\right)=\operatorname{add}\left(\nu P^{\bullet}\right)$. Then there exists a tilting complex $T^{\bullet} \in \mathrm{K}^{\mathrm{b}}\left(\mathcal{P}_{A}\right)$ of length 1 such that

(1) $\operatorname{Hom}_{\mathrm{K}(\operatorname{Mod}-A)}\left(T^{\bullet}, P^{\bullet}[i]\right)=0$ for $i \geq l\left(P^{\bullet}\right)$,

(2) $\operatorname{Hom}_{\mathrm{K}(\operatorname{Mod}-A)}\left(P^{\bullet}[i], T^{\bullet}\right)=0$ for $i<0$, and

(3) $\operatorname{End}_{\mathrm{K}(\operatorname{Mod}-A)}\left(T^{\bullet}\right)$ is a selfinjective artin R-algebra whose Nakayama permutation coincides with that of $A$.

Proof. Set $l=l\left(P^{\bullet}\right)$. We may assume $P^{i}=0$ unless $0 \leq i \leq l$. Note that $\operatorname{add}\left(P^{\bullet}\right)=\operatorname{add}\left(\nu P^{\bullet}\right)$ implies $\operatorname{add}\left(\mathrm{H}^{0}\left(P^{\bullet}\right)\right)=\operatorname{add}\left(\mathrm{H}^{0}\left(\nu P^{\bullet}\right)\right)$. Also, by Lemma 2.4 there exists a torsion theory $(\mathcal{T}, \mathcal{F})$ for mod- $A$ such that $\mathcal{T}={ }^{\perp} \mathrm{H}^{0}\left(P^{\bullet}\right)=$ ${ }^{\perp} \mathrm{H}^{0}\left(\nu P^{\bullet}\right)$ and $\mathcal{F}=\mathcal{T}^{\perp}$. We denote by $t$ the associated torsion radical.

Claim 1: $\mathrm{H}^{l}\left(P^{\bullet}\right) \in \mathcal{T}$ and $\mathrm{H}^{0}\left(P^{\bullet}\right), \mathrm{H}^{0}\left(\nu P^{\bullet}\right) \in \mathcal{F}$.

Proof. By the construction $\mathrm{H}^{0}\left(P^{\bullet}\right), \mathrm{H}^{0}\left(\nu P^{\bullet}\right) \in \mathcal{F}$. Also, by Lemma 1.5

$$
\begin{aligned}
\operatorname{Hom}_{A}\left(\mathrm{H}^{l}\left(P^{\bullet}\right), \mathrm{H}^{0}\left(\nu P^{\bullet}\right)\right) & \simeq \operatorname{Hom}_{\mathrm{K}(\operatorname{Mod}-A)}\left(P^{\bullet}, \nu P^{\bullet}[-l]\right) \\
& \simeq D \operatorname{Hom}_{\mathrm{K}(\operatorname{Mod}-A)}\left(P^{\bullet}, P^{\bullet}[l]\right) \\
& =0
\end{aligned}
$$

and $\mathrm{H}^{l}\left(P^{\bullet}\right) \in \mathcal{T}$.

Claim 2: $\nu: \bmod -A \stackrel{\sim}{\rightarrow} \bmod -A$ induces $\mathcal{T} \stackrel{\sim}{\rightarrow} \mathcal{T}$ and $\mathcal{F} \stackrel{\sim}{\rightarrow} \mathcal{F}$. In particular, $\nu(t(X))=t(\nu X)$ for all $X \in \bmod -A$.

Proof. We have $\nu \mathcal{T}={ }^{\perp}\left(\nu \mathrm{H}^{0}\left(P^{\bullet}\right)\right)={ }^{\perp} \mathrm{H}^{0}\left(\nu P^{\bullet}\right)=\mathcal{T}$ and then $\nu \mathcal{F}=(\nu \mathcal{T})^{\perp}=$ $\mathcal{T}^{\perp}=\mathcal{F}$.

Let $\left\{e_{1}, \cdots, e_{n}\right\}$ be a basic set of orthogonal local idempotents in $A$. Set $I=\{1, \cdots, n\}, I_{1}=\left\{i \in I \mid e_{i} A \in \mathcal{T}\right\}, I_{2}=\left\{i \in I \mid e_{i} A \in \mathcal{F}\right\}$ and $I_{3}=I \backslash I_{1} \cup I_{2}$. For each $i \in I$, we define a complex $T_{i}^{\bullet} \in \mathrm{K}^{\mathrm{b}}\left(\mathcal{P}_{A}\right)$ as follows. Set $T_{i}^{\bullet}=e_{i} A[-1]$ if $i \in I_{1}$, and set $T_{i}^{\bullet}=e_{i} A$ if $i \in I_{2}$. Assume $i \in I_{3}$. Since $e_{i} A$ is indecomposable injective, $t\left(e_{i} A\right)$ is indecomposable. Also, by Lemma 2.6 $t\left(e_{i} A\right)$ is Ext-injective. To this module $t\left(e_{i} A\right)$, we associate an indecomposable Ext-projective module $X_{i} \in \mathcal{T}$ as follows. Set $Y_{1}=t\left(e_{i} A\right)$ and for $k \geq 1$ set $Y_{k+1}=t\left(\tau Y_{k}\right)$ unless $Y_{k}$ is Ext-projective. Then, according to Lemma 2.9, $Y_{m}$ has to be Ext-projective for some $m \geq 1$ because $\mathcal{T}$ contains only a finite number of nonisomorphic indecomposable modules. We set $X_{i}=Y_{m}$ and define $T_{i}^{\bullet}$ as the (-1)-shift of a minimal projective presentation of $X_{i}$. Now, we set $T^{\bullet}=\bigoplus_{i \in I} T_{i}^{\bullet}$ (cf. [12, Theorem 5.8]). Also, we denote by $\rho$ the Nakayama permutation of $A$.

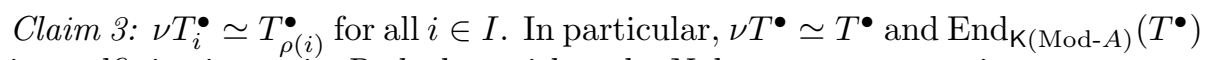
is a selfinjective artin $R$-algebra with $\rho$ the Nakayama permutation. 
Proof. By Claim 2 the sets $I_{i}$ are $\rho$-stable. Thus $\nu T_{i}^{\bullet} \simeq T_{\rho(i)}^{\bullet}$ for $i \in I_{1} \cup I_{2}$. Let $i \in I_{3}$. Then by Claim $2 \nu\left(t\left(e_{i} A\right)\right) \simeq t\left(\nu\left(e_{i} A\right)\right) \simeq t\left(e_{\rho(i)} A\right)$ and hence $\nu X_{i} \simeq X_{\rho(i)}$. Thus $\nu T_{i}^{\bullet} \simeq T_{\rho(i)}^{\bullet}$. Now, for any $i \in I$, by Lemma 1.5

$$
\begin{aligned}
D \operatorname{Hom}_{\mathrm{K}(\operatorname{Mod}-A)}\left(T_{i}^{\bullet}, T^{\bullet}\right) & \simeq \operatorname{Hom}_{\mathrm{K}(\operatorname{Mod}-A)}\left(T^{\bullet}, \nu T_{i}^{\bullet}\right) \\
& \simeq \operatorname{Hom}_{\mathrm{K}(\operatorname{Mod}-A)}\left(T^{\bullet}, T_{\rho(i)}^{\bullet}\right) .
\end{aligned}
$$

Claim 4: $\mathrm{H}^{1}\left(T^{\bullet}\right) \in \mathcal{T}$ and $\mathrm{H}^{0}\left(T^{\bullet}\right), \mathrm{H}^{0}\left(\nu T^{\bullet}\right) \in \mathcal{F}$.

Proof. By the construction $H^{1}\left(T^{\bullet}\right) \in \mathcal{T}$. Also, by Lemma 2.12(1) $\mathrm{H}^{0}\left(\nu T_{i}^{\bullet}\right) \simeq$ $\tau X_{i} \in \mathcal{F}$ for all $i \in I_{3}$ and hence $\mathrm{H}^{0}\left(\nu T^{\bullet}\right) \in \mathcal{F}$. It then follows by Claim 3 that $\mathrm{H}^{0}\left(T^{\bullet}\right) \in \mathcal{F}$.

Claim 5: $T^{\bullet}$ is a tilting complex.

Proof. By Claim $4 \operatorname{Hom}_{\mathrm{K}(\operatorname{Mod}-A)}\left(T^{\bullet}, T^{\bullet}[-1]\right) \simeq \operatorname{Hom}_{A}\left(\mathrm{H}^{1}\left(T^{\bullet}\right), \mathrm{H}^{0}\left(T^{\bullet}\right)\right)=0$. Then by Lemma 1.5 and Claim 3

$$
\begin{aligned}
\operatorname{Hom}_{\mathrm{K}(\operatorname{Mod}-A)}\left(T^{\bullet}, T^{\bullet}[1]\right) & \simeq D \operatorname{Hom}_{\mathrm{K}(\operatorname{Mod}-A)}\left(T^{\bullet}, \nu T^{\bullet}[-1]\right) \\
& \simeq D \operatorname{Hom}_{\mathrm{K}(\operatorname{Mod}-A)}\left(T^{\bullet}, T^{\bullet}[-1]\right) \\
& =0 .
\end{aligned}
$$

Thus by Claim 3 we can apply the last part of Corollary 3.2.

Claim 6: $\operatorname{Hom}_{\mathrm{K}(\operatorname{Mod}-A)}\left(T^{\bullet}, P^{\bullet}[i]\right)=0$ for $i \geq l$ and $\operatorname{Hom}_{\mathrm{K}(\operatorname{Mod}-A)}\left(P^{\bullet}[i], T^{\bullet}\right)=0$ for $i<0$.

Proof. For any $i>l$ we have $a\left(P^{\bullet}[i]\right)<b\left(T^{\bullet}\right)$ and $\operatorname{Hom}_{\mathrm{K}(\operatorname{Mod}-A)}\left(T^{\bullet}, P^{\bullet}[i]\right)=0$. Similarly, for any $i<-1$ we have $a\left(T^{\bullet}\right)<b\left(P^{\bullet}[i]\right)$ and $\operatorname{Hom}_{\mathrm{K}(\operatorname{Mod}-A)}\left(P^{\bullet}[i], T^{\bullet}\right)$ $=0$. Also, by Lemma 1.5 and Claims 1,4

$$
\begin{aligned}
\operatorname{Hom}_{\mathrm{K}(\operatorname{Mod}-A)}\left(T^{\bullet}, P^{\bullet}[l]\right) & \simeq D \operatorname{Hom}_{\mathrm{K}(\operatorname{Mod}-A)}\left(P^{\bullet}, \nu T^{\bullet}[-l]\right) \\
& \simeq D \operatorname{Hom}_{A}\left(\mathrm{H}^{l}\left(P^{\bullet}\right), \mathrm{H}^{0}\left(\nu T^{\bullet}\right)\right) \\
& =0 \\
\operatorname{Hom}_{\mathrm{K}(\operatorname{Mod}-A)}\left(P^{\bullet}[-1], T^{\bullet}\right) & \simeq D \operatorname{Hom}_{\mathrm{K}(\operatorname{Mod}-A)}\left(T^{\bullet}, \nu P^{\bullet}[-1]\right) \\
& \simeq \operatorname{DHom}_{A}\left(\mathrm{H}^{1}\left(T^{\bullet}\right), \mathrm{H}^{0}\left(\nu P^{\bullet}\right)\right) \\
& =0 .
\end{aligned}
$$

This finishes the proof of Lemma 3.4.

Remark 3.5. Consider the case where $l\left(P^{\bullet}\right)=1$ in the above lemma. Then $\operatorname{Hom}_{\mathrm{K}(\operatorname{Mod}-A)}\left(T^{\bullet} \oplus P^{\bullet},\left(T^{\bullet} \oplus P^{\bullet}\right)[i]\right)=0$ for $i \neq 0$ and by [11, Lemma 1.8] we have $P^{\bullet} \in \operatorname{add}\left(T^{\bullet}\right)$. 
Theorem 3.6. Assume $A$ is selfinjective and representation-finite. Let $P^{\bullet} \in$ $\mathrm{K}^{\mathrm{b}}\left(\mathcal{P}_{A}\right)$ be a complex with $\operatorname{Hom}_{\mathrm{K}(\operatorname{Mod}-A)}\left(P^{\bullet}, P^{\bullet}[i]\right)=0$ for $i \neq 0$ and $\operatorname{add}\left(P^{\bullet}\right)=$ $\operatorname{add}\left(\nu P^{\bullet}\right)$. Then there exists some $Q^{\bullet} \in \mathrm{K}^{\mathrm{b}}\left(\mathcal{P}_{A}\right)$ such that $Q^{\bullet} \oplus P^{\bullet}$ is a tilting complex. In particular, if the number of nonisomorphic indecomposable direct summands of $P^{\bullet}$ coincides with the rank of the Grothendieck group $K_{0}(A)$, then $P^{\bullet}$ is a tilting complex.

Proof. Set $l=l\left(P^{\bullet}\right)$. We may assume $P^{i}=0$ unless $0 \leq i \leq l$. In case $l \leq 1$, this is a special case of Corollary 3.2. Assume $l \geq 2$. Let $T^{\bullet} \in \mathrm{K}^{\mathrm{b}}\left(\mathcal{P}_{A}\right)$ be a tilting complex constructed in Lemma 3.4 and set $B=\operatorname{End}_{K(\operatorname{Mod}-A)}\left(T^{\bullet}\right)$. There exists an equivalence of triangulated categories $F: \mathrm{K}^{\mathrm{b}}\left(\mathcal{P}_{A}\right) \stackrel{\sim}{\rightarrow} \mathrm{K}^{\mathrm{b}}\left(\mathcal{P}_{B}\right)$ which sends $T^{\bullet}$ to $B$. Denote by $G: \mathrm{K}^{\mathrm{b}}\left(\mathcal{P}_{B}\right) \stackrel{\sim}{\rightarrow} \mathrm{K}^{\mathrm{b}}\left(\mathcal{P}_{A}\right)$ a quasi-inverse of $F$. Set $\bar{P}^{\bullet}=F\left(P^{\bullet}\right)$. Then $\operatorname{Hom}_{\mathrm{K}(\operatorname{Mod}-B)}\left(\bar{P}^{\bullet}, \bar{P}^{\bullet}[i]\right) \simeq \operatorname{Hom}_{\mathrm{K}(\operatorname{Mod}-A)}\left(P^{\bullet}, P^{\bullet}[i]\right)=0$ for $i \neq 0$. Also, by Lemma $1.8 \nu \bar{P}^{\bullet} \simeq F\left(\nu P^{\bullet}\right)$ and hence $\operatorname{add}\left(\bar{P}^{\bullet}\right)=\operatorname{add}\left(\nu \bar{P}^{\bullet}\right)$. Furthermore,

$$
\begin{aligned}
\mathrm{H}^{i}\left(\bar{P}^{\bullet}\right) & \simeq \operatorname{Hom}_{\mathrm{K}(\operatorname{Mod}-B)}\left(B, \bar{P}^{\bullet}[i]\right) \\
& \simeq \operatorname{Hom}_{\mathrm{K}(\operatorname{Mod}-A)}\left(T^{\bullet}, P^{\bullet}[i]\right) \\
& =0
\end{aligned}
$$

for $i \geq l$ and $\operatorname{Hom}_{\mathrm{K}(\operatorname{Mod}-B)}\left(\bar{P}^{\bullet}[i], B\right) \simeq \operatorname{Hom}_{\mathrm{K}(\operatorname{Mod}-A)}\left(P^{\bullet}[i], T^{\bullet}\right)=0$ for $i<0$, so that $l\left(\bar{P}^{\bullet}\right) \leq l-1$. Thus by induction hypothesis there exists some $\bar{Q}^{\bullet} \in \mathrm{K}^{\mathrm{b}}\left(\mathcal{P}_{B}\right)$ such that $\bar{Q}^{\bullet} \oplus \bar{P}^{\bullet}$ is a tilting complex. Then, by setting $Q^{\bullet}=G\left(\bar{Q}^{\bullet}\right), Q^{\bullet} \oplus P^{\bullet}$ is a tilting complex.

Theorem 3.7. Assume $A$ is selfinjective and representation-finite. Then for any selfinjective artin $R$-algebra $B$ derived equivalent to $A$ the following hold.

(1) There exists a sequence of selfinjective artin $R$-algebras $A=B_{0}, B_{1}, \cdots$, $B_{m}=B$ such that for any $0 \leq i<m, B_{i+1}$ is the endomorphism algebra of a tilting complex for $B_{i}$ of length $\leq 1$.

(2) The Nakayama permutation of $B$ coincides with that of $A$.

Proof. (1) Let $P^{\bullet} \in \mathrm{K}^{\mathrm{b}}\left(\mathcal{P}_{A}\right)$ be a tilting complex with $B \simeq \operatorname{End}_{\mathrm{K}(\operatorname{Mod}-A)}\left(P^{\bullet}\right)$. Set $l=l\left(P^{\bullet}\right)$. In case $l \leq 1$, we have nothing to prove. Assume $l \geq 2$. Let $T^{\bullet} \in \mathrm{K}^{\mathrm{b}}\left(\mathcal{P}_{A}\right)$ be a tilting complex constructed in Lemma 3.4. Set $B_{1}=$ $\operatorname{End}_{\mathrm{K}(\operatorname{Mod}-A)}\left(T^{\bullet}\right)$ and let $F: \mathrm{K}^{\mathrm{b}}\left(\mathcal{P}_{A}\right) \rightarrow \mathrm{K}^{\mathrm{b}}\left(\mathcal{P}_{B_{1}}\right)$ be an equivalence of triangulated categories which sends $T^{\bullet}$ to $B_{1}$. Note that $B_{1}$ is selfinjective and representation-finite, and that $P_{1}^{\bullet}=F\left(P^{\bullet}\right)$ is a tilting complex with $B \simeq$ $\operatorname{End}_{\mathrm{K}\left(\operatorname{Mod}-B_{1}\right)}\left(P_{1}^{\bullet}\right)$. Also, as in the proof of Theorem 3.6, we have $l\left(P_{1}^{\bullet}\right) \leq l-1$. The assertion now follows by induction.

(2) By (1) and Lemma 3.4.

\section{References}

[1] S. Al-Nofayee and J. Rickard, Rigidity of tilting complexes and derived equivalence for self-injective algebras, Preprint. 
[2] M. Auslander, I. Reiten and S. O. Smalø, Representation theory of artin algebras, Cambridge studies in advanced mathematics., 36, Cambridge University Press, 1995.

[3] M. Bökstedt and A. Neeman, Homotopy limits in triangulated categories, Compositio Math. 86 (1993), no. 2, 209-234.

[4] K. Bongartz, Tilted algebras, in: Representations of algebras (Puebla, 1980), 26-38, Lecture Notes in Math., 903, Springer, Berlin.

[5] S. E. Dickson, A torsion theory for Abelian categories, Trans. Amer. Math. Soc. 121 (1966), 223-235.

[6] D. Happel and C. M. Ringel, Tilted algebras, Trans. Amer. Math. Soc. 274 (1982), no. 2, 399-443.

[7] D. Happel, J. Rickard and A. Schofield, Piecewise hereditary algebras, Bull. London Math. Soc. 20 (1988), no. 1, 23-28.

[8] R. Hartshorne, Residues and duality, Lecture Notes in Math., 20, Springer, Berlin, 1966.

[9] M. Hoshino, On splitting torsion theories induced by tilting modules, Comm. Algebra 11 (1983), no. 4, 427-439.

[10] M. Hoshino and Y. Kato, Tilting complexes defined by idempotents, Comm. Algebra 30 (2002), no. 1, 83-100.

[11] M. Hoshino and Y. Kato, Tilting complexes associated with a sequence of idempotents, J. Pure Appl. Algebra 183 (2003), no. 1-3, 105-124.

[12] M. Hoshino, Y. Kato and J.-I. Miyachi, On t-structures and torsion theories induced by compact objects, J. Pure Appl. Algebra 167 (2002), no. $1,15-35$.

[13] Y. Kato, On derived equivalent coherent rings, Comm. Algebra 30 (2002), no. 9, 4437-4454.

[14] S. König and A. Zimmermann, Tilting selfinjective algebras and Gorenstein orders, Quart. J. Math. Oxford Ser. (2) 48 (1997), no. 191, 351-361.

[15] Y. Miyashita, Tilting modules of finite projective dimension, Math. Z. 193 (1986), no. 1, 113-146.

[16] J. Rickard, Morita theory for derived categories, J. London Math. Soc. (2) 39 (1989), no. 3, 436-456.

[17] J. Rickard, Derived categories and stable equivalence, J. Pure Appl. Algebra 61 (1989), 303-317.

[18] J. Rickard, Derived equivalences as derived functors, J. London Math. Soc. (2) 43 (1991), no. 1, 37-48. 
[19] J. L. Verdier, Catégories dérivées, état 0, in: Cohomologie étale, 262311, Lecture Notes in Math., 569, Springer, Berlin, 1977.

Institute of Mathematics, University of Tsukuba, Ibaraki, 305-8571, Japan

E-mail address: abeh@math.tsukuba.ac.jp

Institute of Mathematics, University of Tsukuba, Ibaraki, 305-8571, Japan

E-mail address: hoshino@math.tsukuba.ac.jp 